\title{
Revealing the inherent heterogeneity of human malignancies by variant consensus strategies coupled with cancer clonal analysis
}

\author{
Erich A Peterson, Shweta S Chavan, Michael A Bauer, Christoph J Heuck, Donald J Johann Jr \\ From 11th Annual MCBIOS Conference \\ Stillwater, OK, USA. 6-8 March 2014
}

\begin{abstract}
Tumors are heterogeneous in composition. They are composed of cancer cells proper, along with stromal elements that collectively form a microenvironment, all of which are necessary to nurture the malignant process. In addition, many of the stromal cells are modified to support the unique needs of the malignant state. Tumors are composed of a variety of clones or subpopulations of cancer cells, which may differ in karyotype, growth rate, expression of cell surface markers, sensitivity to therapeutics, etc. New tools and methods to provide an improved understanding of tumor clonal architecture are needed to guide therapy.

The subclonal structure and transcription status of underlying somatic mutations reveal the trajectory of tumor progression in patients with cancer. Approaching the analysis of tumors to reveal clonal complexity in a quantitative manner should facilitate better characterization and therapeutic assignments. The challenge is the interpretation of massive amounts of data from next generation sequencing (NGS) experiments to find what is truly meaningful for improving the understanding of basic cancer biology, as well as therapeutic assignments and outcomes. To meet this need, a methodology named CloneViz was developed and utilized for the identification of serial clonal mutations. Whole exome sequencing (WES) on an Illumina HiSeq 2500 was performed on paired tumor and normal samples from a Multiple Myeloma (MM) patient at presentation, then first and second relapse. Following alignment, a consensus strategy for variant selection was employed along with computational linkage to a formal tumor clonality analysis based on visualization and quantitative methods.
\end{abstract}

\section{Background}

The majority of "cancers" are really a group of diseases, with different molecular signatures. All are characterized by unregulated cell growth and many with the potential to spread and invade other anatomic locations. During the past 15 years there has been tremendous advancement in knowledge of cancer, its molecular nature, characteristics and hallmarks $[1,2]$. The seemingly inherent capability of cancer to adapt dynamically in a Darwinian fashion is a primary reason for therapeutic failures [3]. The landmark paper that established the evolutionary theory of cancer was published by Nowell in 1976 [4], and was based on cytogenetic analysis, which is a whole

\footnotetext{
* Correspondence: don.johann@gmail.com
}

Myeloma Institute for Research and Therapy, University of Arkansas for Medical Sciences, Little Rock, AR, USA genome technique albeit with low resolution by today's standards. It proposed cancer as an evolutionary process driven by stepwise, somatic cell mutations with sequential subclonal selection. Somatic mutations drive the majority of cancers, and many of these are clonal in nature. Through survival advantages these clones become more dominant in the tumor via propagation of progeny by clonal expansion $[5,6]$.

It is established that cancer is a clonal disease that is initiated by a single cell, and the spread of cancer (i.e., metastatic aspects) is also initiated through a single cell $[3,7,8]$. Additionally, it is considered to be a monoclonal disease, that is the cells share the same ancestry and molecular genetics. A subclone is a cell or group of cells, which has formed from an original cell, as a result of a new mutation. Selection events, such as the administration of 
chemotherapy, will kill off some cells and may create mutations in others, conferring resistance to that selection event and a survival advantage. As the clones continue to evolve with new mutations the survival benefits are disseminated among progeny. Many cancers including MM are difficult to cure and treat due to clonal evolution $[3,9,10]$. Perhaps further clonal analysis will provide insights towards therapeutic relapse, resistance, and failure.

MM is an incurable cancer of the bone marrow and is characterized by a malignant proliferation of plasma cells $[11,12]$. Definitive therapies for MM may involve a variety of drugs and approaches and includes two new classes of medications namely, proteasome inhibitors and the immunomodultory drugs (IMiDs) $[13,14]$. Other approaches include autologous tandem transplant [15] and combination chemotherapy. Patient survival has significantly improved over the past 10-15 years but outcomes still vary significantly [16]. An explanation concerning this variation is tumor heterogeneity [17]. Recent genomic sequencing studies have identified somatic mutations in well characterized oncogenes (e.g., $N R A S, K R A S)$ as well as a complex genetic landscape with extensive clonal heterogeneity that serves to limit clinical and scientific utility $[18,19]$. Thus, MM provides a good model for the study of heterogeneity and cancer progression using clonal analysis approaches $[17,20,21]$.

Over time the genetic composition of a tumor may change with different subclones becoming dominant or disappearing. These may occur thru natural selection events due to cell intrinsic or microenvironmental factors [22], or thru selection by therapy. It has been shown that relapse clones were often present via minor subpopulations at diagnosis [23]. Thus, low coverage in the sequencing experiment can also result in missing minor subclones. In this study involving MM clones, what is consistently seen in all time periods is a RAS gene family activation (NRAS, KRAS). This provides an oncogenic signal to the mitogen activated protein kinase (MAPK) pathway leading to uncontrolled cellular growth and survival of that MM clone.

Understanding why tumors progress, especially following what is known to be definitive therapy, is a critical area of research in cancer biology. In this study, the clonal dynamics of a single patient with MM are illustrated by examining three purified bone marrow aspirate samples. Samples were obtained at disease presentation, then first and second relapse. Importantly, a novel bioinformatic approach (toolbox) was developed, which allows for the visualization, quantitation and analysis of the variant/ mutational dynamics and evolution from WES experimental data. The methodology named CloneViz is independent of any cancer type and consists of a suite of computational techniques and analytic methods. MM is used as an illustrated example because of its inherent heterogeneity. Subpopulations of mutations that evolve over time are analyzed. The novelty of the approach concerns the breakdown and analysis of complex WES data sets, deriving a quantitative scrutiny of clonality including aggregate measures, as well as providing a series of interactive visualization techniques, which allow the user/scientist to explore and dissect the clonal dynamics of the experimental datasets.

\section{Methods}

\section{Sample descriptions and library preparation}

Bone marrow aspirates and the peripheral blood sample were collected at the University of Arkansas for Medical Sciences (UAMS), Myeloma Institute for Research and Therapy (MIRT), from a single patient diagnosed with MM. The sample collection protocol was approved by the UAMS Institutional Review Board (IRB). Plasma cells from bone marrow aspirate samples were enriched by anti-CD 138 immuno-magnetic bead selection in a central laboratory as previously described [24]. CD-138 is a marker for a malignant plasma cell, and for all samples used in this study the degree of CD-138 purification was 95\%. The patient's three bone marrow aspirates were obtained at initial presentation (year 2003), first relapse (year 2010) and second relapse (year 2014). Germ line material was obtained from the buffy coat, following density gradient centrifugation of a peripheral blood sample (year 2014). To ensure the absence of plasma cells, buffy coat material was also examined by flow cytometry.

All samples were processed in an identical manner. Whole exome capture libraries were constructed from $100 \mathrm{ng}$ of tumor and normal DNA after shearing, end repair, phosphorylation, and ligation to bar coded sequencing adaptors. DNA was fragmented by the S220 focused-ultrasonicator (Covaris), using a standard protocol for a target bp of 300. DNA was size selected for lengths between $\sim 250$ - $330 \mathrm{bp}$ and subjected to exonic hybrid capture using SeqCap EZ Exome + UTR Library (NimbleGen, Roche). Samples were multiplexed and sequenced on an Illumina HiSeq 2500 using the rapid run mode (paired-end $101 \mathrm{bp}$ reads) to an average depth of coverage of $100 x$, for tumor and normal respectively.

\section{Whole exome sequencing (WES) data analysis and alignment}

Generation of FASTQ files was performed via CASAVA v1.8.2 (http://support.illumina.com/sequencing/sequencing_software/casava.html). Reads were analyzed and quality checked using FastQC (http://www.bioinformatics. babraham.ac.uk/projects/fastqc/). Based on the quality reports, it was decided to trim the last nine bases from each read, using Trimmomatic v0.30 [25]. Paired end reads were aligned to the human genome (GRCh37) by a hybrid approach that utilizes BWA v0.6.2 [26] and Stampy v.1.0.22 [27]. Duplicate reads were marked using Picard 
tools v1.79 (http://picard.sourceforge.net). Sequence recalibration and local realignment were performed using GATK v2.6-4 [28]. Single nucleotide variant (SNV) calling was performed by Strelka v1.0.10 [29], MuTect v1.1.4 [30], and VarScan2 v2.3.6 [31]. Small insertions and deletions (InDels) were called by Strelka and VarScan2. SnpEff v3.5 [32] was used to functionally annotate all variants. Further filtering of variants and comparisons between samples were performed using custom code written in T-SQL, C\#, Perl and R.

Consensus approaches have been used in machine learning to combine findings across multiple methods so that the final rendering of data provides more robust results [33]. This concept was applied to the variant discovery pipeline. Custom software was developed to perform a consensus analysis, utilizing a variety of set-based techniques acting on SNVs and InDels identified by different methods. Consensus SNV analysis processed outputs of Strelka, MuTect, and VarScan2 and also acted on the InDels reported by Strelka and VarScan2. This scrutiny was performed at the level of the variant call frequency (VCF) files, followed by annotation analysis with SnpEff.

The abundance of a variant was determined by computing the product of the variant allelic frequency (VAF), depth (DP) and copy number (CN) [34]. The VAF was determined by dividing the total reads for the variant (TRV) by the sum of the total reads for the variant (TRV) plus total reads for the reference (TRR). Copy number data was derived using ExomeCNV v1.4 [35]. The selection and retention of variants were based on the following filtering parameters: i) $\mathrm{VAF} \geq 10 \%$, and ii) $40 \leq \mathrm{DP} \leq 250$. A manual evaluation of the read alignments using the Integrative Genomics Viewer (IGV) v2.3.32 was also performed [36]. At times a second selection of variants utilized an intersection against a key gene (KG) list. The KG group was constructed from the following public sources: i) known drivers and cancer predisposition genes cited in Vogelstein, et. al. [7], ii) Foundation One Heme ${ }^{\mathrm{TM}}$ Genes (http://foundationone.com/genelist2.php) and, iii) the MD Anderson listing of human DNA repair genes [37].

To quantify clonal diversity in the serial samples of MM diversity measures from ecology were adapted [38]. Each sample is not a single organism/species, but rather consists of thousands of cells from a purified bone marrow aspirate. A particular variant constitutes a molecular species and the abundance is the product of $V A F * D P * C N$. The number of clones in a neoplasm is a simple measure of diversity. Ecological measures of diversity typically integrate both the number and abundance of clones [38]. The Shannon diversity index (SDI) [39] is

$$
S D I=-\sum_{i}^{N} p(i) \ln (p(i))
$$

where $p(i)$ is the frequency of clone $i$ in the neoplasm. The SDI assigns a single quantitative value based on the number of different mutants in the cancer sample, as well as, how evenly distributed each mutant is among the entire group. The SDI value will increase when the number of distinct mutants increases and also when the evenness among the mutants increases [38]. There are other diversity measures (e.g., Simpson's index) but, the Shannon diversity index is preferable because it is not dominated by the most frequent clone, and it has been utilized in previous studies of cancer [40,41].

\section{Results and discussion}

The clonal dynamics of a single patient with MM is illustrated in this study through the analysis of three purified bone marrow aspirates obtained at presentation, then first and second relapses. A bioinformatic approach named CloneViz has been developed to visualize and quantitate the mutational dynamics and evolution of WES data. It has been shown that there is an intraclonal heterogeneity at the level of SNVs (Single Nucleotide Variants) in MM [34]. Clonal analysis has been advocated as a means to study the genetic heterogeneity and branching Darwinian trajectories in cancer [42]. Visualization approaches were developed to provide a "global view" of all mutational events. Unless otherwise stated, all graphics and visualizations in this study were generated by CloneViz.

Additional file 1 displays a genomic mutational overview of the three experiments. A corresponds to the Presentation sample, $\mathbf{B}$ to Relapse \#1 and $\mathbf{C}$ to Relapse \#2. These provide a general view of the inherent mutational events on a chromosomal basis. The $\mathrm{x}$-axis contains an ordered list of chromosomes (1-22, X, Y), each sized by the number of base pairs (bp) it contains. The y-axis is ordered by variant allele frequency (VAF), with color scale indicating sequence depth. Each variant is a point in the plot. Additional file 2 provides the genomic mutational overview in a tabular format. Variant counts are tallied and grouped by chromosome across the three experiments.

Additional file 3 shows two paired scatter plots involving the three samples. Plot A displays the Presentation on the $\mathrm{x}$-axis versus Relapse \#1 on y-axis. A color assignment is used to discriminate paired variants (blue) from individual (green for Presentation, and red for Relapse \#1). Note, overlays result in darker colors. Both the $x$ and $y$-axes are based on VAF. Plot $\mathbf{B}$ uses the same conventions. These plots also provide an overall sense of the mutational landscape; in this case, what is shared and different between the three samples. Generally, observed are a significant portion of shared mutations but also a fair number of differences.

CloneViz contains interactive tools, but more importantly is a bioinformatic approach, with a variety of 
options allowing for the visual exploration and evolutionary/Darwinian analysis of NGS variant data. Figure 1 shows a series of Gaussian kernel density plots with associated scatter plots indicating the frequency of the cells carrying all acquired mutations. The peaks in the kernel density plot may indicate dominant clones and any subclones. The clonal populations are visualized by calculating the percent of mutant variant reads for all acquired mutations in the sample and adjusting for $\mathrm{CN}$. This generates a frequency of mutated cells for each variant [34].

Key genes (KG) are labelled on the scatter plots. The $\mathrm{x}$-axis for both plots is VAF. The $\mathrm{y}$-axis for the kernel density plot is the density function. The scatter plot $\mathrm{y}$-axis is the product of copy number $(\mathrm{CN})$ and depth. Each sphere on the scatter plot represents a variant. As spheres overlap the color is darker. Figure 1, subfigure A corresponds to the Presentation sample, B is Relapse $\# 1$ and $\mathbf{C}$ is Relapse \#2. The kernel density plots show several distinct peaks in all three samples indicating a level of heterogeneity across samples.

Additional file 4 shows a series of kernel density plots with associated scatter plots for all three samples and are only based on the key genes. Plot A corresponds to the Presentation sample, B to Relapse \#1 and $\mathbf{C}$ to Relapse \#2. Distinct peaks are seen in all three samples indicating heterogeneity. Additional file 5 shows a third type of CloneViz generated plot series. Here the kernel density is linked to a series of individual scatter plots based on copy number (color coded). The $\mathrm{x}$-axis of all graphs represents the tumor VAF and y-axis the sequence depth. This representation of the data follows the SciClone approach [43], and A corresponds to Presentation, B Relapse \#1 and C Relapse \#2.

Figure 2 illustrates the variant dynamics and clonal evolution across the three samples and table 1 provides a tabular view. Both are an extension of the CloneViz analyses. The Presentation sample was obtained when the patient was initially diagnosed with MM, contains 132 variants/mutants that passed filtering criteria, and was found to have a Shannon Diversity Index (SDI) of 4.67. The six mutants from the key genes group in this sample are ARHGAP26, ARID1A, ERBB4, PARP1, ATM, and the NRAS oncogene.

Incorporating the findings from Figure 1 plot $\mathbf{A}$, additional file 4 plot $\mathbf{A}$, additional file 5 plot $\mathbf{A}$, and table 1 it is evident that NRAS, which has CN of two, and VAF of 44 can be considered to be a significant member of
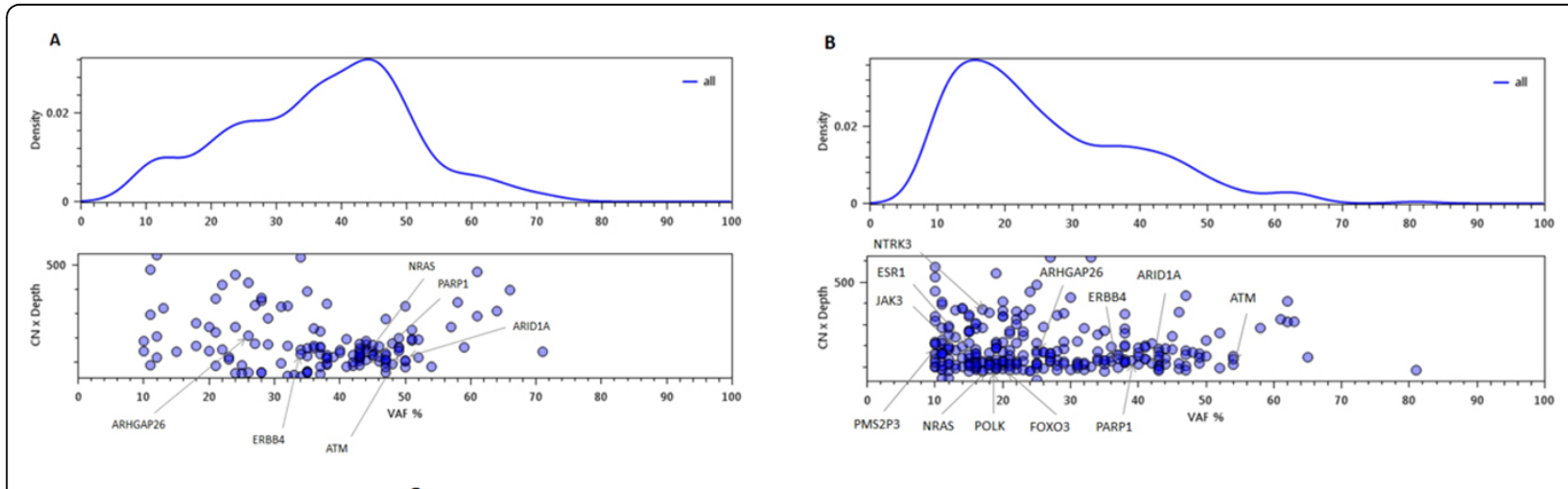

C
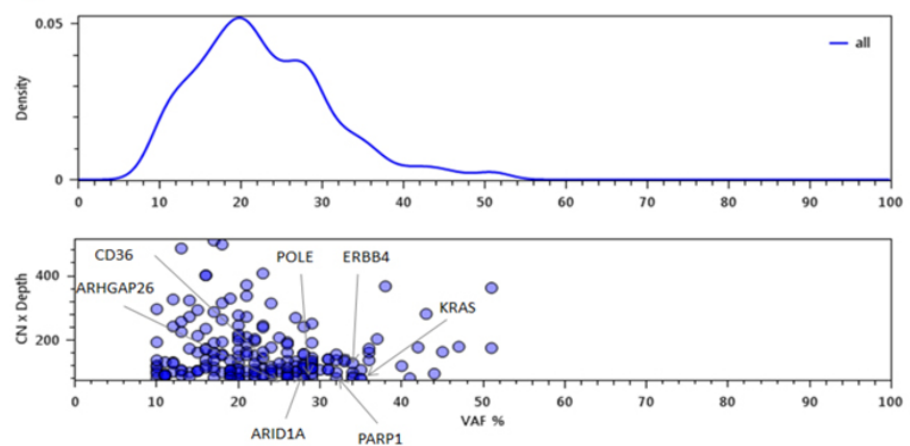

Figure 1 Kernel density and scatter plot of all variants. Gaussian kernel densities with associated scatter plots indicating the frequency of the cells carrying all acquired mutations for the three samples are listed. A denotes the Presentation sample, B Relapse \#1 and C Relapse \#2. The peaks in the kernel density plot may indicate dominant clones and any subclones. The x-axis for both kernel density and scatter plots are VAF. The $y$-axis in each density plot is density (adjusted by copy number), and the $y$-axis in each scatter plot is $C N$ * Depth. Key genes are annotated. 


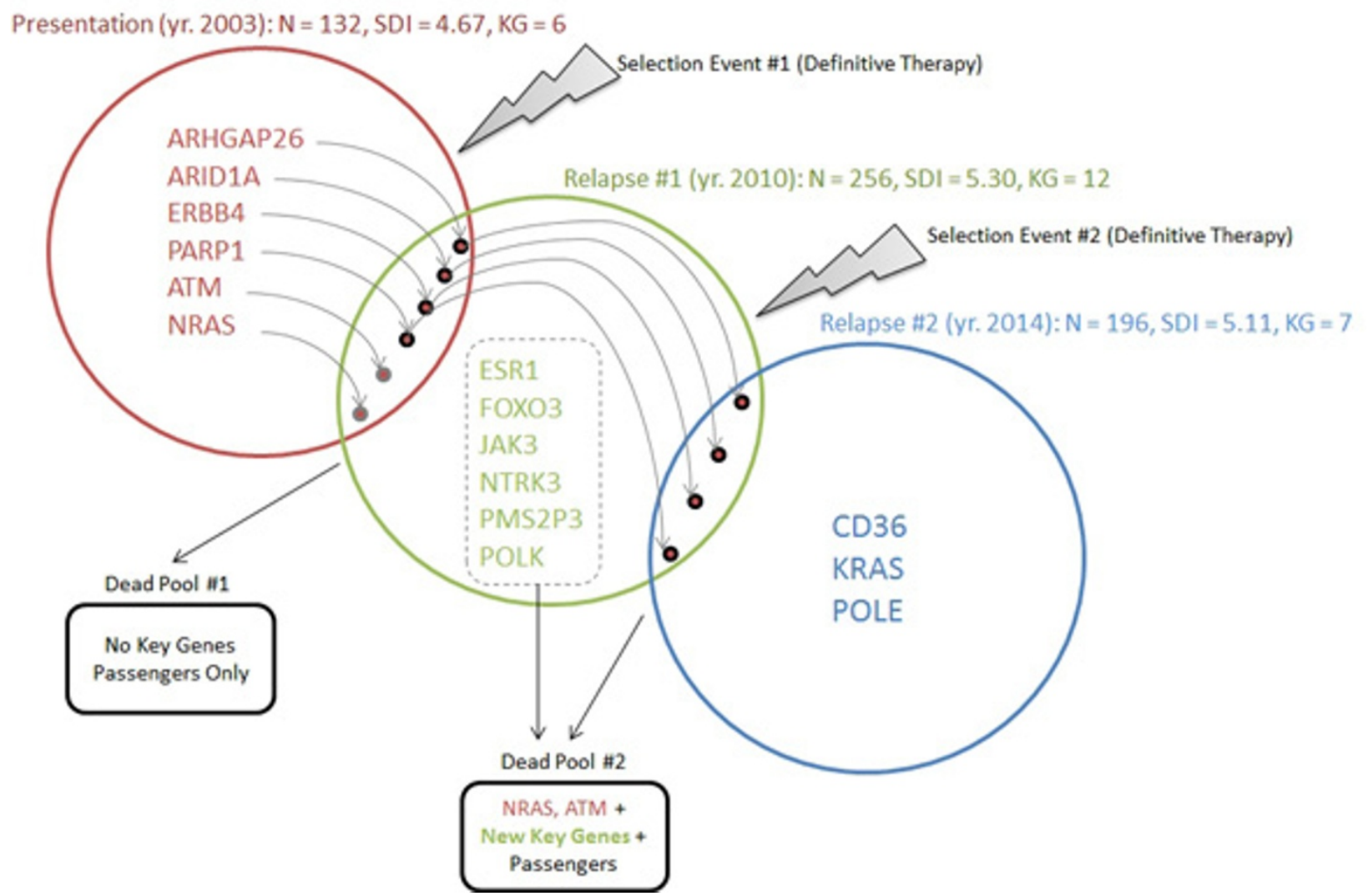

Figure 2 Variant dynamics and clonal evolution. The variant dynamics and clonal evolution of key genes across the Presentation (red), Relapse \#1 (green) and Relapse \#2 (blue) samples are illustrated. Information regarding the year each sample was obtained, total number of mutations $(\mathrm{N})$, computed Shannon Diversity Index (SDI), and the number of key genes (KG) are provided for each sample. Key genes identified within each sample are listed. An arc from a gene symbol into another sample indicates the survival of the mutation through the selection event (definitive therapy). "Dead Pools" list key genes and/or passengers that do not survive.

Table 1 Tabular view of variant dynamics and clonal evolution.

\begin{tabular}{|c|c|c|c|c|c|c|c|c|c|c|}
\hline \multirow[b]{2}{*}{$\begin{array}{l}\text { Gene } \\
\text { Symbol }\end{array}$} & \multirow[b]{2}{*}{$\begin{array}{l}\text { Func. } \\
\text { Class }\end{array}$} & \multicolumn{3}{|c|}{ Presentation } & \multicolumn{3}{|c|}{ Relapse \#1 } & \multicolumn{3}{|c|}{ Relapse \#2 } \\
\hline & & $\begin{array}{l}\text { VAF } \\
\%\end{array}$ & $\mathrm{CN}$ & $\mathrm{DP}$ & $\begin{array}{l}\text { VAF } \\
\%\end{array}$ & $\mathrm{CN}$ & DP & $\begin{array}{l}\text { VAF } \\
\%\end{array}$ & $\mathrm{CN}$ & DP \\
\hline ARHGAP26 & VUS & 26 & 3 & 70 & 25 & 3 & 59 & 26 & 2 & 46 \\
\hline ARID1A & TSG & 50 & 2 & 52 & 44 & 2 & 57 & 28 & 2 & 43 \\
\hline ATM & TSG & 49 & 3 & 49 & 54 & 3 & 46 & NP & NP & NP \\
\hline$\overline{C D 36}$ & VUS & NP & $\mathrm{NP}$ & NP & $\mathrm{NP}$ & $\mathrm{NP}$ & $\mathrm{NP}$ & 23 & 2 & 73 \\
\hline ERBB4 & VUS & 34 & 2 & 76 & 37 & 2 & 79 & 32 & 2 & 50 \\
\hline ESR1 & VUS & $N P$ & NP & NP & 15 & 2 & 107 & $\mathrm{NP}$ & $\mathrm{NP}$ & NP \\
\hline FOXO3 & VUS & NP & $\mathrm{NP}$ & NP & 21 & 2 & 68 & NP & $\mathrm{NP}$ & NP \\
\hline JAK3 & OG & $\mathrm{NP}$ & $\mathrm{NP}$ & $N P$ & 12 & 3 & 66 & $\mathrm{NP}$ & $\mathrm{NP}$ & NP \\
\hline KRAS & OG & NP & NP & NP & NP & NP & NP & 35 & 2 & 40 \\
\hline NRAS & OG & 44 & 2 & 87 & 19 & 1 & 91 & NP & $\mathrm{NP}$ & NP \\
\hline NTRK3 & VUS & NP & $\mathrm{NP}$ & NP & 18 & 3 & 119 & $\mathrm{NP}$ & $\mathrm{NP}$ & NP \\
\hline PARP1 & DR & 45 & 2 & 65 & 39 & 2 & 66 & 32 & 2 & 41 \\
\hline PMS2P3 & DR & NP & $\mathrm{NP}$ & $\mathrm{NP}$ & 11 & 3 & 76 & $\mathrm{NP}$ & $\mathrm{NP}$ & NP \\
\hline$\overline{\text { POLE }}$ & DR & $\mathrm{NP}$ & $\mathrm{NP}$ & $\mathrm{NP}$ & $\mathrm{NP}$ & $\mathrm{NP}$ & $\mathrm{NP}$ & 29 & 2 & 48 \\
\hline POLK & DR & NP & NP & NP & 19 & 2 & 58 & $\mathrm{NP}$ & $\mathrm{NP}$ & NP \\
\hline
\end{tabular}

A tabular form of the clonal dynamics as shown in figure 2 illustrates changes in VAF, copy number, and depth across the Presentation, Relapse \#1 and Relapse \#2 samples. Abbreviations: CN (Copy Number), DP (Depth), DR (DNA Repair), NP (Not Present), OG (Oncogene), VAF (Variant Allele Frequency), VUS (Variant of Uncertain Significance) and TSG (Tumor Suppressor Gene). 
the dominant/founder clone for the Presentation sample. This is further strengthened by the non-synonymous hotspot mutation involving codon 13, where glycine is replace by arginine (additional file 6 p.Gly13Arg/ c.37G $>C$ ). In most tumor types exhibiting a mutation of a RAS gene family member (HRAS, KRAS, NRAS), the mutational activation of one member predominates (e.g., KRAS in lung and colon cancer). However, MM belongs to a subset of cancers that mutate multiple RAS family members, and it has been observed that mutations in one family member are mutually exclusive to mutations in other family members [44]. Historically, it's been reported that there are relatively high and equal rates of $N R A S$ and KRAS mutations that are $\sim 20 \%$ respectively in MM [45]. Recently, the large MM genomic sequencing studies have identified mutations in the MAPK pathway affecting KRAS (23\%) and NRAS (20\%), and have shown these aberrations to play a significant role $[18,19]$. Under physiologic conditions, the MAPK pathway tightly regulates cellular functions such as growth, differentiation and survival. Mutations resulting in constitutive activation of NRAS or KRAS cause a persistent activation of downstream mediators in the MAPK pathway. It is suspected that each RAS family member provides a similar oncogenic signal to the MAPK pathway [46].

Selection event \#1 (Figure 2) occurs later in year 2003, as a result of the patient receiving definitive therapy for MM. The patient did well until year 2010 when the first relapse occurred and a new bone marrow aspirate was obtained. Comparing the Presentation vs. Relapse \#1 samples reveals that all of the key genes survive, including the $N R A S$ oncogene. In addition, the JAK3 oncogene is gained along with two mutated DNA repair genes ( $P M S 2 P 3$ and $P O L K)$. Dead Pool \#1 contains only passengers (non-key genes). The term "Dead Pool" indicates mutations lost in the evolution of the cancer. The sample from Relapse \#1 contains 256 variants/mutants and an increased SDI (5.30), from Presentation. With an information theory view, this indicates more uncertainty or randomness in the process. With a cancer biology view, this indicates a progression/diversification in the mutational landscape. This sample contains mutants from 12 key genes and includes two oncogenes (NRAS and JAK3).

Why did the cancer recur and what mutation(s) are the likely culprit(s)? Examining table 1 and additional file 6 the oncogenic mutation in NRAS is the same, although it now has a $\mathrm{CN}$ of one and VAF of 19. The relative abundance of this mutation has been diminished but it is still present and still driving the MAPK pathway and malignant cellular proliferation. Incorporating the findings from Figure 1 plot $\mathbf{B}$, additional file 4 plot $\mathbf{B}$, additional file 5 plot $\mathbf{B}$, and table 1 it is evident that NRAS can be considered to be a significant member of the dominant clone for the Relapse \#1 sample. The JAK3 mutation is noted to be "downstream"/intronic and without involvement in splice sites. Hence, it is much less likely to directly affect protein function and is not considered as a major offender in the cancer recurrence.

Selection event \#2 (Figure 2) occurs later in year 2010 as a result of the patient receiving a second round of definitive therapy to address the relapsed MM. The patient did well until year 2014 when a second relapse occurred and a new bone marrow aspirate was obtained. Comparison of Relapse \#1 to Relapse \#2, shows that both mutants and passengers die, and Dead Pool \#2 contains eight key genes (NRAS, ATM, ESR1, FOXO3, JAK3, NTRK3, PMS2P3, POLK) and passengers. The sample from Relapse \#2 contains 196 variants/mutants, with a SDI of 5.11, which is slightly less than Relapse \#1 but still higher than Presentation. It also contains mutants from 7 key genes (ARHGAP26*, ARID1A*, ERBB4\%, PARP1*, CD36, KRAS, POLE). Four mutants (denoted by *) have survived since Presentation. Although the NRAS and JAK3 oncogenes are lost, the KRAS oncogene is gained. KRAS contains a non-synonymous hotspot mutation involving codon 61, where glutamine is replace by histadine (additional file $6 \mathrm{p}$. Gln61His/c.183A $>$ C). Incorporating the findings from Figure 1 plot $\mathbf{C}$, additional file 4 plot $\mathbf{C}$, additional file 5 plot $\mathbf{C}$, and table 1 it appears that KRAS can be considered to be an important subclone for the Relapse \#2 sample. Despite the fact that NRAS has been lost, the mutational activation of KRAS provides a new RAS subclone, which has a $\mathrm{CN}$ of 2 and VAF of 35, and able to drive the MAPK pathway, thus continuing the aberrant cellular proliferation signals from this malignant subclone. Additional file 7 lists general tallies/statistics for the variant effect types for the three samples.

Why was there another recurrence? First, MM is currently not curable and the vast majority of patients will recur, albeit at different time intervals. Second, although in each instance a definitive therapy approach was taken and the patient did have a remission, none of the two MM care regimens addressed the mutation in the RAS gene family member. Examination of the CN, VAF and clonality visualizations, makes it apparent that the hotspot mutated RAS species was quite viable. Mutated RAS species are known to have a similar oncogenic signal and in each instance there was a sufficient abundance to drive the MAPK pathway. Although there was a remission in each case, the mutated RAS species was never fully eliminated, and provided uninterrupted aberrant proliferation signals from year 2003 to 2014.

There is now a new drug named Trametinib, for tumors with activated MAPK pathways due to hotspot mutated KRAS or NRAS species. This is contained in additional file 8 which lists the potential therapeutics for the various key genes from the Drug Gene Interaction 
database (DGIdb) [47]. Definitive therapy can now include this new agent and better target the source of the deviant proliferation signals. For cancer treatment scenarios where there have been relapses following definitive therapy and/or the standard-of-care options are poor, drug assignments based on the mutational landscape of the particular patient's cancer may provide benefit, and are an active research topic in clinical trials and translational medicine [48].

\section{Conclusions}

Understanding why tumors progress, especially following what is thought to be definitive therapy, is a fundamental topic in cancer biology. This study illustrated an analysis of the clonal dynamics of a single patient with MM, by examining three purified bone marrow aspirate samples obtained at disease presentation, first and second relapse using a custom bioinformatic approach and methodology named CloneViz. The approach allowed for the visualization and quantitation of the variant/mutational dynamics and evolution from WES. Subpopulations of mutations will evolve over time due to natural selection events related to cell intrinsic or microenvironmental factors, and also as a function of therapeutically induced selection events. These serve to eliminate some mutations/variants, but may also provide a survival advantage for others.

The demonstrated novelty of CloneViz concerns the breakdown and analysis of complex WES data sets. It performs clonal analysis, which has been advocated as a means to study the genetic heterogeneity and branching Darwinian trajectories found in many cancers, which currently limits aggregate approaches for scientific and clinical utility. A temporal-based quantitative examination of clonality from serial MM samples was performed that included individual as well as aggregate measures, and provided a series of interactive visualizations, allowing the user to explore and dissect the clonal dynamics. Observed in all serial MM samples was the presence of a RAS gene species (NRAS, KRAS) with a hotspot mutation, known to provide a similar oncogenic signal/activation of the MAPK pathway promoting aberrant cellular proliferation. A permanent remission or cure was not achieved despite definitive therapy. It was unlikely that therapy would produce a cure or lasting remission since the dominant genetic alterations in the founder clone and emerging secondary clone were never targeted specifically for therapy. To make major advances in cancer therapy, a systematic approach to collect tissue samples at diagnosis, and serially at relapse(s) in order to profile the dynamic clonal evolution is critical and sorely needed [49].

It is understood that cancer is a clonal disease that is initiated by a single cell. Additionally cancer metastasis, which is the spread of the disease from the primary site, is also initiated through a single cell, and importantly, is the chief reason many patients with cancer die. The seemingly inherent capability of cancer to adapt dynamically in a Darwinian fashion is a primary reason for therapeutic failures. Survival advantages occur as a result of intrinsic cell and microenvironmental factors as well as cancer therapies. These selected, "more fit" clones are then able to "out compete" their competition and become more dominant in the tumor via propagation of their progeny by clonal expansion, leading to relapse, therapeutic resistance and eventually death. Bioinformatic approaches addressing clonality and consensus strategies for the analysis and improved understanding of the complex cancer genetic landscape are needed, and the methodologies illustrated in CloneViz represent movement in this direction.

\section{Additional material}

\begin{abstract}
Additional file 1: Genomic mutational overview. A genomic mutational overview of the three experiments is computed and displayed. A corresponds to the Presentation sample, B to Relapse \#1 and C to Relapse \#2. These provide a general view of the inherent mutational events on a chromosomal basis. The $x$-axis contains an ordered list of chromosomes $(1-22, X, Y)$, each sized by the number of base pairs (bp) it contains. The $y$-axis is ordered by variant allele frequency (VAF), and the color scale indicates sequence depth. Each variant is a point in the plot.
\end{abstract}

Additional file 2: Genomic mutational overview (tabular format). Variant counts are tallied and grouped by chromosome across the three experiments.

Additional file 3: Scatter plots of paired samples. This exploratory analysis begins to illustrate what is shared and different between the three samples. A displays variants in the Presentation on the $\mathrm{x}$-axis compared to Relapse \#1 on y-axis and B shows variants in Relapse \#1 on the $x$-axis compared to Relapse $\# 2$ on the $y$-axis. Both the $x$ and $y$-axes are based on VAF. Variants are colored to indicate whether they are shared or unique. See legend for color assignments.

Additional file 4: Kernel density and scatter plot of key genes. Kerne densities with associated scatter plots based only on key genes is shown for all samples. A denotes the Presentation sample, B Relapse \#1 and C Relapse \#2. Axes and conventions are the same as in Figure 1.

Additional file 5: Kernel density and scatter plot of all variants discriminated by copy number. Kernel density and associated scatter plots that include all mutations are shown for each of the three samples. In each subfigure the kernel density and scatter plot is further separated by copy number (color coded, see legend). For each graph, the $x$-axis represents the tumor VAF and $y$-axis the sequence depth. A denotes the Presentation sample, B Relapse \#1 and C Relapse \#2.

Additional file 6: Key genes mutational data. Mutational data for key genes is listed across the Presentation, Relapse \#1 and Relapse \#2 samples. Listed for each key gene mutation are variant types, as well as, predicted amino acid protein change, cDNA change (in HGVS notation) for nonsynonymous coding, and stop gained effect types. Abbreviation: NP (Not Present).

Additional file 7: Summary of variant effect types. Tabular list and summary of the general variant effects for the Presentation, Relapse \#1, and Relapse \#2 samples.

Additional file 8: Key gene therapeutics from the drug gene interaction database (DGldb). Tabular listing of potential therapeutics for the key genes obtained from the Drug Gene Interaction database (DGldb) 


\section{Authors' contributions}

DJJ and EAP conceived and designed the study. EAP, MAB, SSC, CJH and DJJ performed experiments and analyses. DJJ and EAP designed the software. EAP and MAB implemented the software. DJJ and EAP wrote the manuscript. All authors approved the manuscript.

\section{Declarations}

Publication of this work was supported by Program Project Grant CA 55813, from the National Institutes of Health $(\mathrm{NIH})$, National Cancer Institute (NCl), Bethesda, MD, USA.

This article has been published as part of BMC Bioinformatics Volume 15 Supplement 11, 2014: Proceedings of the 11th Annual MCBIOS Conference. The full contents of the supplement are available online at http://www. biomedcentral.com/bmcbioinformatics/supplements/15/S11.

\section{Published: 21 October 2014}

\section{References}

1. Hanahan D, Weinberg RA: The hallmarks of cancer. Cell 2000, 100(1):57-70.

2. Hanahan D, Weinberg RA: Hallmarks of cancer: the next generation. Cell 2011, 144(5):646-674

3. Greaves M, Maley CC: Clonal evolution in cancer. Nature 2012, 481(7381):306-313

4. Nowell PC: The clonal evolution of tumor cell populations. Science 1976, 194(4260):23-28.

5. Welch JS, Ley TJ, Link DC, Miller CA, Larson DE, Koboldt DC, Wartman LD, Lamprecht TL, Liu F, Xia J, et al: The origin and evolution of mutations in acute myeloid leukemia. Cell 2012, 150(2):264-278.

6. Gerlinger M, Swanton C: How Darwinian models inform therapeutic failure initiated by clonal heterogeneity in cancer medicine. $\mathrm{Br} J$ Cancer 2010, 103(8):1139-1143.

7. Vogelstein B, Papadopoulos N, Velculescu VE, Zhou S, Diaz LA, Kinzler KW: Cancer genome landscapes. Science 2013, 339(6127):1546-1558.

8. Potter NE, Ermini L, Papaemmanuil E, Cazzaniga G, Vijayaraghavan G, Titley I, Ford A, Campbell P, Kearney L, Greaves M: Single-cell mutational profiling and clonal phylogeny in cancer. Genome Res 2013, 23(12):2115-2125.

9. Greaves M: Darwinian medicine: a case for cancer. Nat Rev Cancer 2007, 7(3):213-221

10. Stratton MR: Exploring the genomes of cancer cells: progress and promise. Science 2011, 331(6024):1553-1558.

11. Kyle RA, Rajkumar SV: Multiple myeloma. N Engl J Med 2004, 351(18):1860-1873.

12. Rajkumar SV: Treatment of multiple myeloma. Nat Rev Clin Oncol 2011, 8(8):479-491

13. Singhal S, Mehta J, Desikan R, Ayers D, Roberson P, Eddlemon P, Munshi N, Anaissie E, Wilson C, Dhodapkar M, et al: Antitumor activity of thalidomide in refractory multiple myeloma. N Engl J Med 1999, 341(21):1565-1571.

14. Hideshima T, Richardson P, Chauhan D, Palombella VJ, Elliott PJ, Adams J, Anderson KC: The proteasome inhibitor PS-341 inhibits growth, induces apoptosis, and overcomes drug resistance in human multiple myeloma cells. Cancer Res 2001, 61(7):3071-3076.

15. Barlogie B, Jagannath S, Desikan KR, Mattox S, Vesole D, Siegel D, Tricot G, Munshi N, Fassas A, Singhal S, et al: Total therapy with tandem transplants for newly diagnosed multiple myeloma. Blood 1999 93(1):55-65.

16. Kumar SK, Rajkumar SV, Dispenzieri A, Lacy MQ, Hayman SR, Buadi FK, Zeldenrust SR, Dingli D, Russell SJ, Lust JA, et al: Improved survival in multiple myeloma and the impact of novel therapies. Blood 2008, 111(5):2516-2520.

17. Morgan GJ, Walker BA, Davies FE: The genetic architecture of multiple myeloma. Nat Rev Cancer 2012, 12(5):335-348.

18. Lohr JG, Stojanov P, Carter SL, Cruz-Gordillo P, Lawrence MS, Auclair D, Sougnez C, Knoechel B, Gould J, Saksena G, et al: Widespread genetic heterogeneity in multiple myeloma: implications for targeted therapy. Cancer Cell 2014, 25(1):91-101.

19. Chapman MA, Lawrence MS, Keats JJ, Cibulskis K, Sougnez C, Schinzel AC, Harview CL, Brunet JP, Ahmann GJ, Adli M, et al: Initial genome sequencing and analysis of multiple myeloma. Nature 2011, 471(7339):467-472.

20. Walker BA, Wardell CP, Melchor L, Brioli A, Johnson DC, Kaiser MF, Mirabella F, Lopez-Corral L, Humphray S, Murray L, et al: Intraclonal heterogeneity is a critical early event in the development of myeloma and precedes the development of clinical symptoms. Leukemia 2014, 28(2):384-390.

21. Keats JJ, Chesi M, Egan JB, Garbitt VM, Palmer SE, Braggio E, Van Wier S, Blackburn PR, Baker AS, Dispenzieri A, et al: Clonal competition with alternating dominance in multiple myeloma. Blood 2012, 120(5):1067-1076.

22. Mbeunkui F, Johann DJ Jr: Cancer and the tumor microenvironment: a review of an essential relationship. Cancer Chemother Pharmacol 2009, 63(4):571-582.

23. Mullighan CG, Phillips LA, Su X, Ma J, Miller CB, Shurtleff SA, Downing JR: Genomic analysis of the clonal origins of relapsed acute lymphoblastic leukemia. Science 2008, 322(5906):1377-1380.

24. Zhan F, Hardin J, Kordsmeier B, Bumm K, Zheng M, Tian E, Sanderson R, Yang $Y$, Wilson $C$, Zangari $M$, et al: Global gene expression profiling of multiple myeloma, monoclonal gammopathy of undetermined significance, and normal bone marrow plasma cells. Blood 2002, 99(5):1745-1757.

25. Bolger AM, Lohse M, Usadel B: Trimmomatic: A flexible trimmer for Illumina Sequence Data. Bioinformatics 2014.

26. Li H, Durbin R: Fast and accurate short read alignment with BurrowsWheeler transform. Bioinformatics 2009, 25(14):1754-1760.

27. Lunter G, Goodson M: Stampy: a statistical algorithm for sensitive and fast mapping of Illumina sequence reads. Genome Res 2011, 21(6):936-939.

28. DePristo MA, Banks E, Poplin R, Garimella KV, Maguire JR, Hartl C, Philippakis AA, del Angel G, Rivas MA, Hanna M, et al: A framework for variation discovery and genotyping using next-generation DNA sequencing data. Nat Genet 2011, 43(5):491-498.

29. Saunders CT, Wong WS, Swamy S, Becq J, Murray LJ, Cheetham RK: Strelka: accurate somatic small-variant calling from sequenced tumor-normal sample pairs. Bioinformatics 2012, 28(14):1811-1817.

30. Cibulskis K, Lawrence MS, Carter SL, Sivachenko A, Jaffe D, Sougnez C, Gabriel S, Meyerson M, Lander ES, Getz G: Sensitive detection of somatic point mutations in impure and heterogeneous cancer samples. Nat Biotechnol 2013, 31(3):213-219.

31. Koboldt DC, Zhang Q, Larson DE, Shen D, McLellan MD, Lin L, Miller CA, Mardis ER, Ding L, Wilson RK: VarScan 2: somatic mutation and copy number alteration discovery in cancer by exome sequencing. Genome Res 2012, 22(3):568-576.

32. Cingolani P, Platts A, Wang le L, Coon M, Nguyen T, Wang L, Land SJ, Lu X, Ruden DM: A program for annotating and predicting the effects of single nucleotide polymorphisms, SnpEff: SNPs in the genome of Drosophila melanogaster strain w1118; iso-2; iso-3. Fly (Austin) 2012, 6(2):80-92.

33. Dietterich TG: Ensemble methods in machine learning. Springer; 2000.

34. Walker BA, Wardell CP, Melchor L, Hulkki S, Potter NE, Johnson DC, Fenwick K, Kozarewa I, Gonzalez D, Lord CJ, et al: Intraclonal heterogeneity and distinct molecular mechanisms characterize the development of $t$ $(4 ; 14)$ and $\mathrm{t}(11 ; 14)$ myeloma. Blood 2012, 120(5):1077-1086.

35. Sathirapongsasuti JF, Lee H, Horst BA, Brunner G, Cochran AJ, Binder S, Quackenbush J, Nelson SF: Exome sequencing-based copy-number variation and loss of heterozygosity detection: ExomeCNV. Bioinformatics 2011, 27(19):2648-2654.

36. Thorvaldsdottir H, Robinson JT, Mesirov JP: Integrative Genomics Viewer (IGV): high-performance genomics data visualization and exploration. Briefings in bioinformatics 2013, 14(2):178-192.

37. [http://sciencepark.mdanderson.org/labs/wood/dna_repair_genes. html\#Human].

38. Magurran A: Measuring biological diversity Malden: Blackwell; 2004

39. Shannon C: A mathematical theory of communication. The Bell System Technical Journal 1948, 27:379-423.

40. Almendro V, Kim HJ, Cheng YK, Gonen M, Itzkovitz S, Argani P, van Oudenaarden A, Sukumar S, Michor F, Polyak K: Genetic and phenotypic diversity in breast tumor metastases. Cancer Res 2014, 74(5):1338-1348.

41. Maley CC, Galipeau PC, Finley JC, Wongsurawat VJ, Li X, Sanchez CA, Paulson TG, Blount PL, Risques RA, Rabinovitch PS, et al: Genetic clonal diversity predicts progression to esophageal adenocarcinoma. Nat Genet 2006, 38(4):468-473.

42. Burgess DJ: Cancer genetics: Initially complex, always heterogeneous. Nat Rev Cancer 2011, 11(3):153. 
43. Kandoth C, McLellan MD, Vandin F, Ye K, Niu B, Lu C, Xie M, Zhang Q McMichael JF, Wyczalkowski MA, et al: Mutational landscape and significance across 12 major cancer types. Nature 2013, 502(7471):333-339

44. Fernandez-Medarde A, Santos E: Ras in cancer and developmental diseases. Genes Cancer 2011, 2(3):344-358.

45. Bezieau S, Devilder MC, Avet-Loiseau H, Mellerin MP, Puthier D, Pennarun E, Rapp MJ, Harousseau JL, Moisan JP, Bataille R: High incidence of $\mathrm{N}$ and $\mathrm{K}$-Ras activating mutations in multiple myeloma and primary plasma cell leukemia at diagnosis. Hum Mutat 2001, 18(3):212-224.

46. Mulligan G, Lichter DI, Di Bacco A, Blakemore SJ, Berger A, Koenig E, Bernard H, Trepicchio W, Li B, Neuwirth R, et al: Mutation of NRAS but not KRAS significantly reduces myeloma sensitivity to single-agent bortezomib therapy. Blood 2014, 123(5):632-639.

47. Griffith M, Griffith OL, Coffman AC, Weible JV, McMichael JF, Spies NC, Koval J, Das I, Callaway MB, Eldred JM, et al: DGldb: mining the druggable genome. Nat Methods 2013, 10(12):1209-1210.

48. Mirnezami R, Nicholson J, Darzi A: Preparing for precision medicine. $N$ Engl J Med 2012, 366(6):489-491.

49. Govindan R, Ding L, Griffith M, Subramanian J, Dees ND, Kanchi KL, Maher CA, Fulton R, Fulton L, Wallis J, et al: Genomic landscape of nonsmall cell lung cancer in smokers and never-smokers. Cell 2012, 150(6):1121-1134.

doi:10.1186/1471-2105-15-S11-S9

Cite this article as: Peterson et al:: Revealing the inherent heterogeneity of human malignancies by variant consensus strategies coupled with cancer clonal analysis. BMC Bioinformatics 2014 15(Suppl 11):S9.

\section{Submit your next manuscript to BioMed Central} and take full advantage of:

- Convenient online submission

- Thorough peer review

- No space constraints or color figure charges

- Immediate publication on acceptance

- Inclusion in PubMed, CAS, Scopus and Google Scholar

- Research which is freely available for redistribution

Submit your manuscript at www.biomedcentral.com/submit
Biomed Central 